Rapid Reviews COVID-19•

\title{
Review 1: "Neutralization against B.1.351 and B.1.617.2 with sera of COVID-19 recovered cases and vaccinees of BBV152"
}

\author{
Nathaniel Roy Landau' ${ }^{1}$
}

${ }^{1}$ NYU Langone Medical Center

Published on: Aug 23, 2021

License: Creative Commons Attribution 4.0 International License (CC-BY 4.0). 


\section{$\underline{\text { RR:C19 Evidence Scale rating by reviewer: }}$}

- Reliable. The main study claims are generally justified by its methods and data. The results and conclusions are likely to be similar to the hypothetical ideal study. There are some minor caveats or limitations, but they would/do not change the major claims of the study. The study provides sufficient strength of evidence on its own that its main claims should be considered actionable, with some room for future revision.

$* * * * * * * * * * * * * * * * * * * * * * * * * * * * * * * * * * * * * * *$

\section{Review:}

The Yadav et al. report focuses on the antibody response to immunization with the BBV152 vaccination. BBV152 (aka Covaxin) is an inactivated SARS-CoV-2 vaccine consisting of chemically inactivated virions with an added TLR7/8 adjuvant. The vaccine, based on the earlier Wuhan isolate, has been previously shown to be effective against viruses with the earlier D614G spike protein. In this very brief report, the researchers test the antibodies in the sera of vaccinated individuals for neutralization of the D614G, Beta, and Delta viruses. The investigators find that the titer of antibodies drops only slightly (about 3-fold). Furthermore, the titer of the antibodies the vaccine elicits is similar to the titers found in convalescent patients. Thus, the vaccine is predicted to be effective, even though these titers are significantly less than expected for mRNA vaccines.

This is a very brief report consisting of one figure. The data appears to be statistically significant, and the findings are helpful to support the effectiveness of the vaccine against the variants. The vaccine is in use in India, where the prevalence of the Delta variant is high; therefore, it is important to understand vaccine effectiveness there. In the final sentence of the paper, the authors state that the inactivated virus vaccine has broad epitope coverage. The intent of this particular sentence is unclear because other vaccines in use are expected to induce an equally broad antibody response against the spike protein. Perhaps the authors intend to say that the vaccine could potentially invoke a $\mathrm{T}$ cell response to the viral nucleocapsid. This response is absent from the mRNA, adeno, and recombinant spike protein vaccines. The $\mathrm{T}$ cell response would be broader because the other vaccines encode only the spike protein. 
The authors declare no financial interests; however, two authors are affiliated with Bharat Biotech, the company that manufactures the vaccine. The authors should acknowledge this in the conflict of interest statement. 